\title{
Pervasive Cryptic Epistasis in Molecular Evolution
}

\author{
Mark Lunzer ${ }^{1}$, G. Brian Golding ${ }^{2}$, Antony M. Dean ${ }^{1,3,4 *}$
}

1 BioTechnology Institute, University of Minnesota, St. Paul, Minnesota, United States of America, 2 Department of Biology, McMaster University, Hamilton, Ontario, Canada, 3 Department of Ecology, Evolution and Behavior, University of Minnesota, St. Paul, Minnesota, United States of America, 4 Instituto Gulbenkian de Ciência, Oeiras, Portugal

\begin{abstract}
The functional effects of most amino acid replacements accumulated during molecular evolution are unknown, because most are not observed naturally and the possible combinations are too numerous. We created 168 single mutations in wildtype Escherichia coli isopropymalate dehydrogenase (IMDH) that match the differences found in wild-type Pseudomonas aeruginosa IMDH. 104 mutant enzymes performed similarly to E. coli wild-type IMDH, one was functionally enhanced, and 63 were functionally compromised. The transition from $E$. coli $\mathrm{IMDH}$, or an ancestral form, to the functional wild-type $P$. aeruginosa IMDH requires extensive epistasis to ameliorate the combined effects of the deleterious mutations. This result stands in marked contrast with a basic assumption of molecular phylogenetics, that sites in sequences evolve independently of each other. Residues that affect function are scattered haphazardly throughout the IMDH structure. We screened for compensatory mutations at three sites, all of which lie near the active site and all of which are among the least active mutants. No compensatory mutations were found at two sites indicating that a single site may engage in compound epistatic interactions. One complete and three partial compensatory mutations of the third site are remote and lie in a different domain. This demonstrates that epistatic interactions can occur between distant $(>20 \AA)$ sites. Phylogenetic analysis shows that incompatible mutations were fixed in different lineages.
\end{abstract}

Citation: Lunzer M, Golding GB, Dean AM (2010) Pervasive Cryptic Epistasis in Molecular Evolution. PLoS Genet 6(10): e1001162. doi:10.1371/ journal.pgen.1001162

Editor: David S. Guttman, University of Toronto, Canada

Received June 13, 2010; Accepted September 16, 2010; Published October 21, 2010

Copyright: (c) 2010 Lunzer et al. This is an open-access article distributed under the terms of the Creative Commons Attribution License, which permits unrestricted use, distribution, and reproduction in any medium, provided the original author and source are credited.

Funding: Research supported by NIH grant GM060611 and funds from UMN. The funders had no role in study design, data collection and analysis, decision to publish, or preparation of the manuscript.

Competing Interests: The authors have declared that no competing interests exist.

* E-mail: deanx024@umn.edu

\section{Introduction}

In a half century of molecular phylogenetics there never has been a systematic investigation of the functional and fitness effects of amino acid replacements in evolution. Experimental studies focus on those few mutations that change protein function [1]. Of the remaining thousands of replacements nothing is said - they may or may not be of functional consequence. Sequence analyses use statistical approaches to explore modes of evolution [2,3]. Rarely does fitting alternative evolutionary models to observed data disallow alternative explanations. For example, to some [4-6] an elevated ratio of amino acid replacements to silent substitutions between species $\left(d_{n} / d_{s}\right)$ suggests evidence for the action of positive selection. To others [7] it suggests relaxed selection against slightly deleterious amino acid replacements during population bottlenecks. Both interpretations are viable.

Non-additive interactions among mutations (epistasis) are critical to protein structure and function $[1,8]$ and consequently to speciation [9], the evolution of sex [10], recombination [11], dominance [12], robustness [13] and human disease [14]. Nonadditive interactions force sites to functionally co-vary during evolution $[15,16]$. Computational methods that ignore phylogenetic structure [17-29] fail to distinguish between co-variation arising from functional causes and co-variation arising though shared common ancestry. The latter, an ineluctable product of shared history, is reflected in the bifurcating hierarchy of a phylogenetic tree (a tree must collapse to a star burst if no sites covary). Computational methods that account for phylogenetic structure [30-38] have identified sites likely to functionally coevolve $[32,36]$. The relative scarcity of such sites accords with the observation that most amino acid replacements occur at the surfaces of proteins where solvent exposed side chains are less likely to interact $[39,40]$. On the other hand it may simply reflect a lack of statistical power in many, though not all [32], of computational methods used. An alternative approach identifies pathogenic missense mutations in one species that have no obvious detrimental effect in a related species $[41,42]$. This approach does not detect deleterious mutations of minor phenotypic effect.

Computationally derived predictions need empirical verification [1]. Gloor et al. [43] used site directed mutagenesis to confirm the epistasis predicted between co-evolving residues in yeast phosphoglycerate kinase. Experiments with yeast iso-2-cytochrome $c$ [44] also identified epistatic interactions between sites. However, two other studies, one with game bird lysozymes $[45,46]$ and one with vertebrate p53 domains [47], failed to find any evidence of epistasis. Several other site directed mutagenesis studies identified epistatic interactions among positively selected replacements in TEM-1 $\beta$-lacatamase [48], vertebrate steroid receptors [49] and visual pigments [50] and in coral red fluorescent proteins [51]. In no case, however, have experiments been designed to explore the prevalence of epistasis in molecular evolution in general.

Here, we explore the prevalence of epistasis in molecular evolution from the distribution of functional effects caused by individual mutations introduced to one sequence from a homologue in another species. We studied the leuB encoded $\beta$ isopropylmalate dehydrogenase (IMDH) because: 1) the enzyme 


\section{Author Summary}

Many bioinformatics and functional genomics predictions are derived from evolutionary patterns of amino acid replacement in protein sequence alignments. Most computational methods assume that replacements in one sequence will be tolerated in all related sequences. Here, we evaluate-by direct experiment-the functional impact of amino acid replacements accumulated during the course of evolution. Our initial results show that cryptic interactions among amino acid replacements are common and that most are deleterious. This result has implications not only for the evolution of function, recombination, sex, dominance, robustness, disease, and even speciation, but also for practical applications-in conservation biology (e.g. to decide which organisms to preserve) and in vaccine design (e.g. using consensus or reconstructed ancestral sequences). Analyzing one interaction in detail, we find that compensatory mutations need not lie in close proximity to the original mutation as generally supposed. This result suggests that unsuspected structure-function relationships can be revealed by analyzing patterns of siteto-site interactions among amino acid replacements in evolution.

has a conserved well defined role in leucine biosynthesis [52,53]; 2) high resolution x-ray crystallography of divergent IMDHs $(<35 \%$ identical) reveals a conserved protein fold [54-57]; sequence alignments show that divergent IMDHs rarely differ by more than a few insertions and deletions; and 4) the relationship between enzyme performance $\left(k_{\text {cat }} / K_{m . \mathcal{N A D}}\right)$ and fitness has been determined using Escherichia coli as a model system [58]. The IMDHs from two mesophyles, E. coli and P. aeruginosa, differ at 168 of 365 sites including six small indels (Figure S1) located in flexible loops external to the core structure. Conserved in fold and function, $E$. coli and $P$. aeruginosa IMDHs provide excellent material with which to investigate protein evolution arising through sequence divergence in the absence of major changes in structure and function.

\section{Results/Discussion}

\section{Functional Effects of Single Amino Acid Replacements}

We constructed 168 site directed mutants of E. coli leuB (each with a single mutation from $P$. aeruginosa leuB) and then expressed and purified each enzyme and determined its kinetic parameters. The resulting distribution of enzyme performances $\left(k_{c a t} / K_{m . \mathcal{N} A D}\right)$ is strongly skewed to the left and only a single outlier with increased performance lies on the right (Figure 1A). The error distribution, obtained by repeatedly assaying wild-type E. coli IMDH, is Gaussian, $\bar{x}=1, s d=0.09$ (Figure 1B). This same distribution is expected of amino acid replacements that do not affect function. The 52 mutants with relative performances above $E$. coli wild-type form a half-Gaussian distribution, $\bar{x}=1, s d=0.08$, similar to the error distribution. This suggests $168=2 \times 52=104$ mutations have no detectable effect on enzyme performance, 63 reduce it, and one increases it. Pair-wise $t$-tests (for unequal replication and unequal variances [59]) combine with a $5 \%$ false discovery rate [60] to identify 61 mutants of changed performance: 56 have decreased performance and 5 have increased performance, with only the single outlier having performance increased by more than $15 \%$.

The assumption that mutations act independently, and hence additively, leads to a predicted performance for $P$. aeruginosa IMDH that is clearly wrong. The sum of the individual mutational effects and the $E$. coli $k_{\text {cat }} / K_{m . N A D}$ is negative: $k_{c a t} / K_{m . N A D}=$ $-1874 \mathrm{mM}^{-1} \mathrm{sec}^{-1}$. This is a physical impossibility. The assump-

A
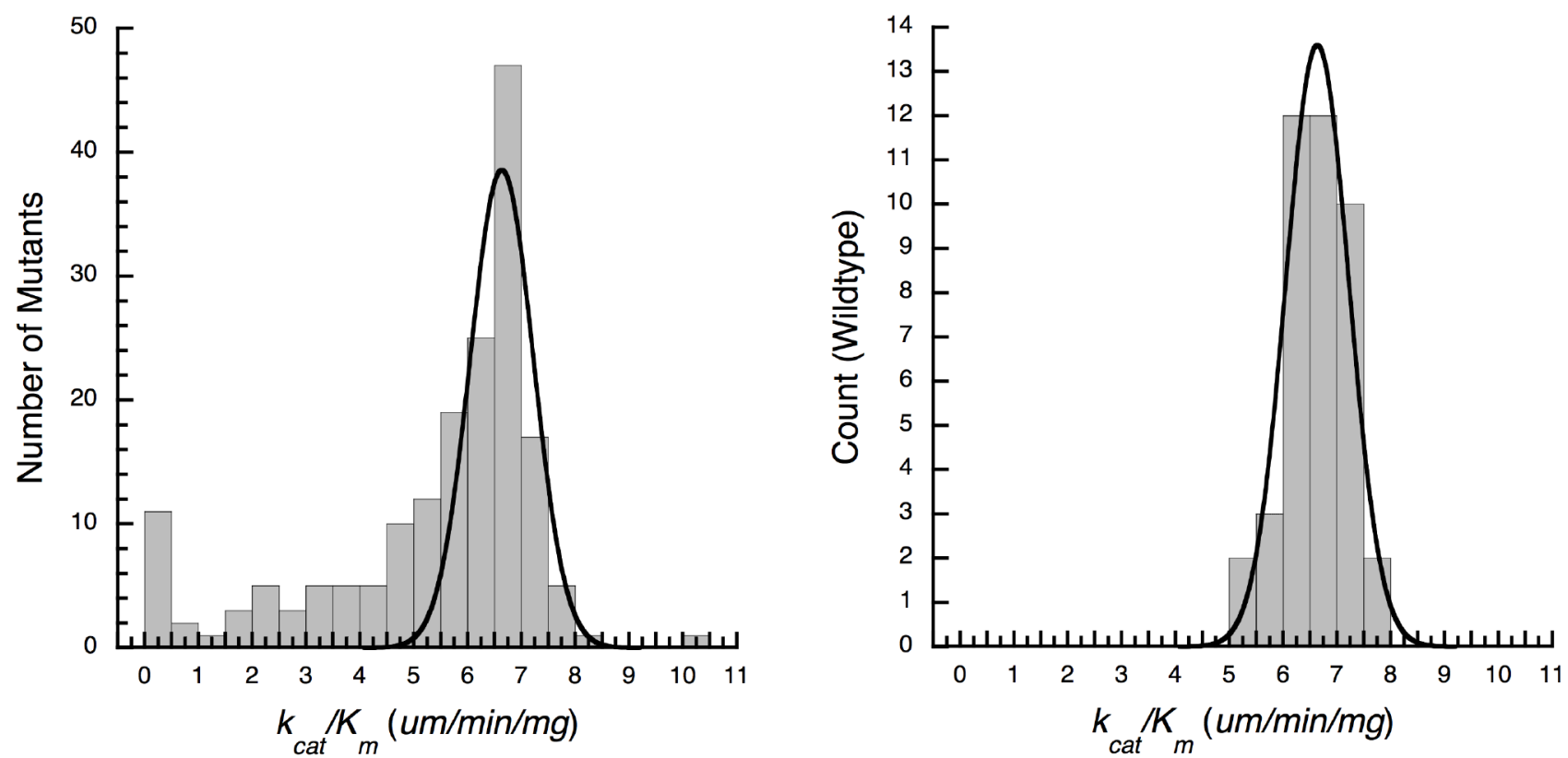

Figure 1. Cryptic epistasis in IMDH. (A) Distribution of functional effects produced upon moving single amino acids and indels from $P$. aeruginosa IMDH into E. coli IMDH. The shoulder on the left indicates the presence of extensive epistasis. (B) Error distribution obtained by repeatedly assaying wild-type E. coli IMDH. Gaussian distributions fitted as described in the text.

doi:10.1371/journal.pgen.1001162.g001 
tion that mutations act multiplicatively is also wrong. In simple transition state theory $k_{\text {cat }} / K_{m . N A D} \propto e^{-\Delta G^{\prime} / R T}$, where $\Delta G^{\prime}$ is the difference in free energy between the ground state and the transition state, $R$ is the gas constant and $T$ is ${ }^{\circ}$ Kelvin [61]. The difference in free energy between the transition states of the mutant and wild-type enzymes is $\Delta \Delta G^{\prime} \propto \log _{e}\left(k_{\mathrm{cat}}^{\mathrm{mutant}} / K_{\mathrm{m} . \mathrm{NAD}}^{\mathrm{mutant}}\right)-\log _{e}\left(k_{\mathrm{cat}}^{\mathrm{Ec}} / K_{\mathrm{m} . \mathrm{NAD}}^{\mathrm{Ec}}\right)$. With five mutants completely inactive the sum of the $\Delta \Delta G^{\prime}$ and $\log _{e}\left(k_{\mathrm{cat}}^{\mathrm{Ec}} / K_{\mathrm{m} \text {.NAD }}^{\mathrm{Ec}}\right)$ is minus infinity and the predicted performance is $k_{\text {cat }} / K_{m . N A D}=0$. In fact $P$. aeruginosa IMDH has a $k_{\text {cat }} / K_{m . N A D}=42.8 \pm 1.3 \mathrm{mM}^{-1} \mathrm{sec}^{-1}, n=10$, slightly lower than that of $E$. coli IMDH which has a $k_{\text {cat }} / K_{m . N A D}=$ $66.4 \pm 0.9 \mathrm{mM}^{-1} \mathrm{sec}^{-1}, n=41$. The inescapable conclusion is that amino acid replacements at many sites interact. IMDH evolution is characterized by rampant epistasis that remains cryptic until revealed by experiment.

That only 64 of the 168 sites affect function certainly underestimates the number that interact epistatically. To understand this, consider two cases in which two sites are involved in a simple pair-wise interaction (Figure 2). In each case, only one of the two sites reduces function when an amino acid in one species is mutated to that found in another. Mutating the second site restores the ancestral functional state. Mutations at both sites may reduce function if three or more amino acid replacements arose during the course of evolution (Figure S2A) - although this is not guaranteed. In a simple network of pair-wise interactions only two of three sites might be identified (Figure S2B). We expect some of the remaining 104 sites to engage in epistatic interactions.

Zuckerkandl [15] first proposed that amino acid replacements at one site in a protein might influence the acceptability of amino acid replacements at other sites. Fitch and Markowitz [16] suggested that as species diverge from a common ancestor their sets of variable sites also diverge to explore different regions of sequence space. Mutating a currently invariant site in one species by introducing an amino acid from a homologous protein in another species risks producing a loss-of-function mutant. The many functionally compromised mutants in this study amply confirm the insight of these early pioneers.

\section{Location of Deleterious Amino Acid Replacements}

Mutations affecting function ( $<80 \%$ wild-type performance) are scattered throughout the IMDH structure (Figure S3). Solvent accessibility, distance to the catalytic center (Asp 251), secondary structure and rate of amino acid replacement per site do not correlate significantly with performance for the mutants analyzed here. Only one mutation, F73L, likely affects catalysis by contacting a substrate directly (Figure S4).

\section{Identifying Compensatory Mutations}

We screened for compensatory mutations of F73L, A94D and A284C, all of which lie near the active site and all of which are among the least active mutants. We combined each deleterious mutation with each of the 167 remaining mutations, expressed and purified each double mutant, and assayed their activities. Four mutations compensate the F73L mutation (Table 1). F120A, identified in the original screen because it produces a $50 \%$ increase in wild-type performance, now produces a 6 -fold increase in performance so that the F73L,F120A double mutant, while not as active as the $E$. coli wild-type enzyme, is as active as the $P$. aeruginosa enzyme. Three other compensatory mutants, F132L C136I and I179V, do not individually affect wild-type performance. Performance is completely restored to $E$. coli wild-type levels in the F73L,I179V double mutant. Performance is partly restored in the F73L,F132L and F73L,C136I double mutants. No mutations were found to restore function to A94D and A284C.
A

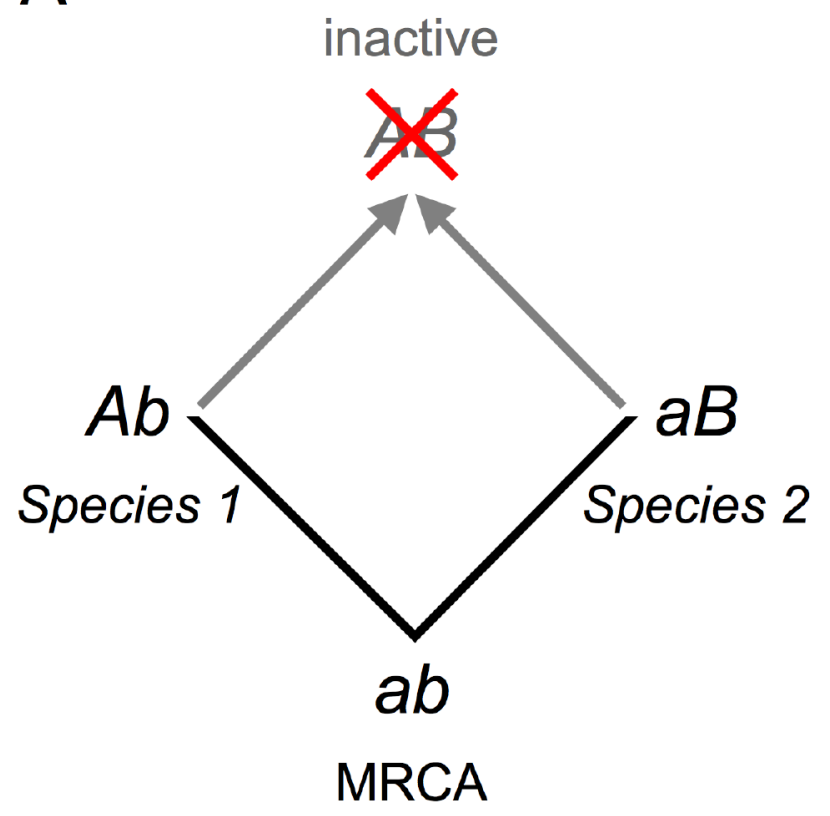

B

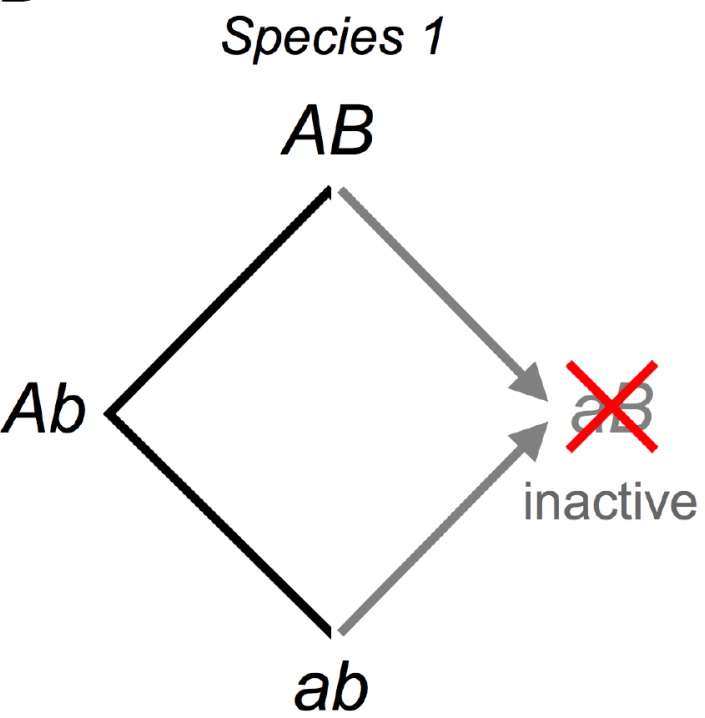

Species 2

Figure 2. The evolution of cryptic epistasis. (A) Two lineages diverge from the most recent common ancestor (MRCA), genotype $a b$. Each fixes a mutation to produce genotypes $A b$ and $a B$. The presence of either mutation prevents the second becoming fixed because the double mutant, genotype $A B$, is deleterious. Cryptic epistasis is revealed upon moving mutation $A$ from species 1 into species 2 or moving mutation $B$ from species 2 into species 1 (gray arrows). Moving mutations $a$ or $b$ to form genotype $a b$ restores the ancestral functional state. (B) Cryptic epistasis can also occur when both mutations arise in the same lineage leading to species 1. In this case cryptic epistasis is revealed upon moving mutation $a$ into species 1 or mutation $B$ into species 2 (gray arrows). Moving mutations $A$ or $b$ to form genotype $A b$ regenerates a functional evolutionary intermediate. doi:10.1371/journal.pgen.1001162.g002 
Table 1. Performance of mutants towards NAD.

\begin{tabular}{lll}
\hline & \multicolumn{2}{l}{$\begin{array}{l}\text { Performance* } \\
\boldsymbol{k}_{\text {cat }} / \boldsymbol{K}_{\text {m.NAD }}\left(\mathbf{m M}^{-\mathbf{1}} \mathbf{s e c}^{-\mathbf{1}}\right)\end{array}$} \\
\cline { 2 - 3 } & Mutant & F73L \\
\cline { 2 - 3 } Enzyme & $\mathbf{F 7 3}$ & 7 \\
\hline E. coli wt & 166 & 44 \\
F120A & 102 & 25 \\
F132L & 182 & 39 \\
C136I & 172 & 66 \\
I179V & 161 & 42 \\
P. aeruginosa wt & & \\
\hline *All standard errors $<10 \%$ of the estimates. & \\
doi:10.1371/journal.pgen.1001162.t001
\end{tabular}

Our results are compatible with several types of interactions. Using E. coli IMDH performance as a standard suggests a simple pair-wise interaction between L73 and I179 because only L73F and $\mathrm{I} 179 \mathrm{~V}$ are fully compensatory. Using the lower $P$. aeruginosa IMDH performance as a standard suggests a high-order interaction with function compromised only when residues L73, F120, C136, I179 are combined. Replacing any one amino acid, L73F, F120A, C136I, or I179V destroys the 4-way interaction to restore full performance. That no mutation restores function to A94D and A284C demonstrates several compensatory mutations are essential; at least two sites $(A$ and $B)$ must each interact with each original mutation $(X)$ to form a simple chain $(A-X-B)$.

Whereas previous experimental studies [41-45] assumed interacting residues would be in close physical contact, all compensatory mutations for $\mathrm{F} 73 \mathrm{~L}$ in the large domain lie more than $20 \AA$ distant in the small domain, close to a hinge in the bsheet on which the two domains swivel (Figure 3). This suggests a common mode of action, possibly related to repositioning the F73L-shifted nicotinamide ring for catalysis. Our experimental strategy, of moving replacements from one homologue into another and screening for compensatory mutations, is useful in that it provides a general means to identify interacting sites regardless of the mechanisms involved.

\section{Predicted Fitness Effects}

The predicted fitness effects of most mutations are tiny. Previous work [56] established that wild-type E. coli IMDH lies on a fitness plateau (Figure 4A). In this limit of adaptation [62], increases in performance do not improve fitness and even large reductions in IMDH performance produce small fitness effects (Figure 4B). Indeed, $65 \%$ of the selection coefficients are predicted to be less than $10^{-5} /$ generation. Selection during starvation growth with glucose as the sole limiting resource is far greater than in nature where leucine is both widely available and abundant [63]. Many mutations, including the one with increased performance, are likely selectively neutral, or very nearly so.

\section{Two Models of Neutral Evolution}

The cryptic epistasis we revealed is consistent with two modes of neutral evolution: the covarion process [64] and the nearly neutral process [65]. In the covarion process, neutral and/or beneficial mutations are fixed in different lineages that, when brought together in the same protein, are deleterious (Figures 2, Figure S2). In the nearly neutral process successive slightly deleterious alleles are fixed by random genetic drift (particularly during population
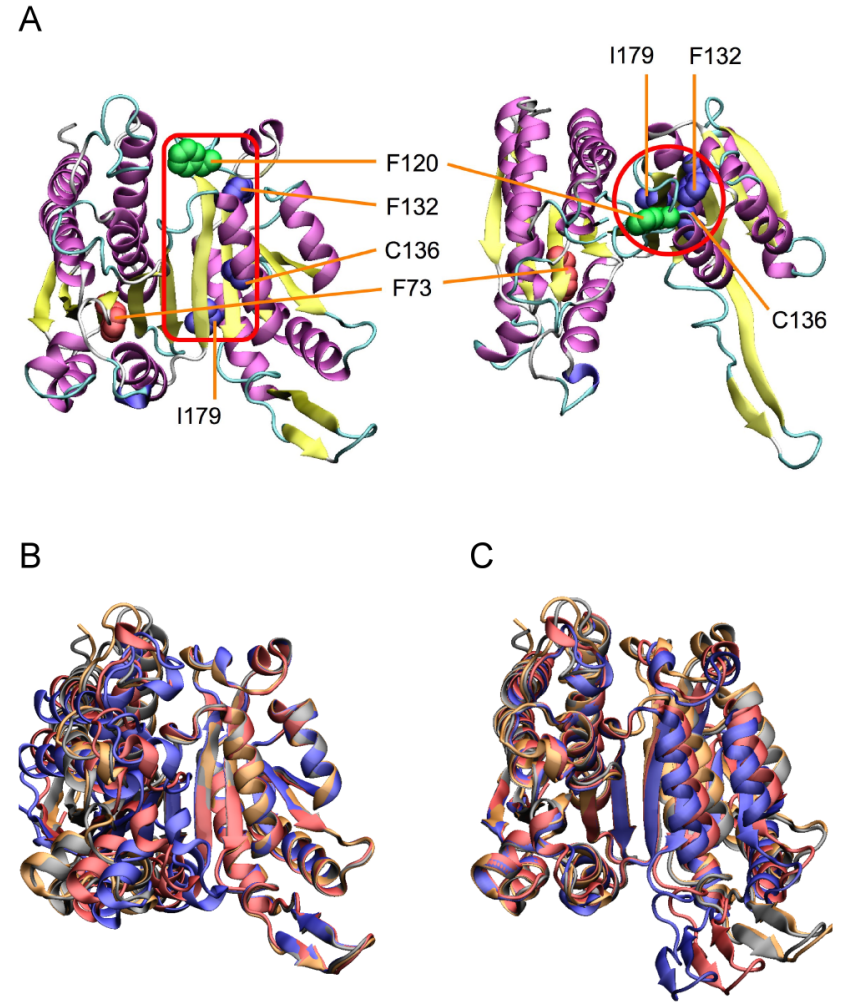

Figure 3. Locations of compensatory mutations for F73L. Compensatory mutations for the deleterious effects of F73L in the large domain are located in the small domain close to the hinge in the b-sheet. (A) Two views of the locations of the compensatory mutations for F73L in E. coli IMDH (space filling: brown for F73L, green for F120A and blue for F132L, C136I and I179V). (B) The two domains of IMDH are built on the $\beta$-sheet and move as rigid bodies. Superimposing the small right-hand domains of $E$. coli and $S$. typhimurium IMDHs reveals rigid body movements of the large domains on the left. (C) Superimposing the large left-hand domains reveals rigid body movements of the small domains on the right. The hinge region lies in the $\beta$-sheet between the two domains. Structures from E. coli (pdb 1CM7) and S. typhimurium IMDHs (pdb 1CNZ).

doi:10.1371/journal.pgen.1001162.g003

bottlenecks) until a compensatory mutation arises that, on restoring full activity, is fixed by positive selection (particularly after a population expands). The concave fitness function for E. coli IMDH (Figure S4), typical of dominance curves [12], provides the fitness plateau on which fitness could gradually drift downwards as slightly deleterious mutations sequentially fix before a beneficial compensatory mutation restores full activity.

The two processes can be distinguished by determining the order in which mutations arise during the course of evolution. Phylogenetic analysis suggests that the most recent common ancestor (MRCA) of $E$. coli and $P$. aeruginosa had amino acids FAFVV at sites 73, 120, 132, 136 and 179 (Figure 5). On the lineage leading to E. coli mutations V136C and V179I arose first (the order is indeterminate) before mutation A120F. Each mutation is compatible with F73. On the lineage leading to $P$. aeruginosa mutations $\mathrm{F} 73 \mathrm{~L}$ and $\mathrm{F} 132 \mathrm{~V}$ arose (the order is indeterminate) before mutation V132L and finally mutation V136I. The presence of V179 is expected to compensate the potentially deleterious interaction between L73 and F132 in the event that the F73L mutation arose first. Hence, the pattern of replacements supports the nearly neutral process because a 

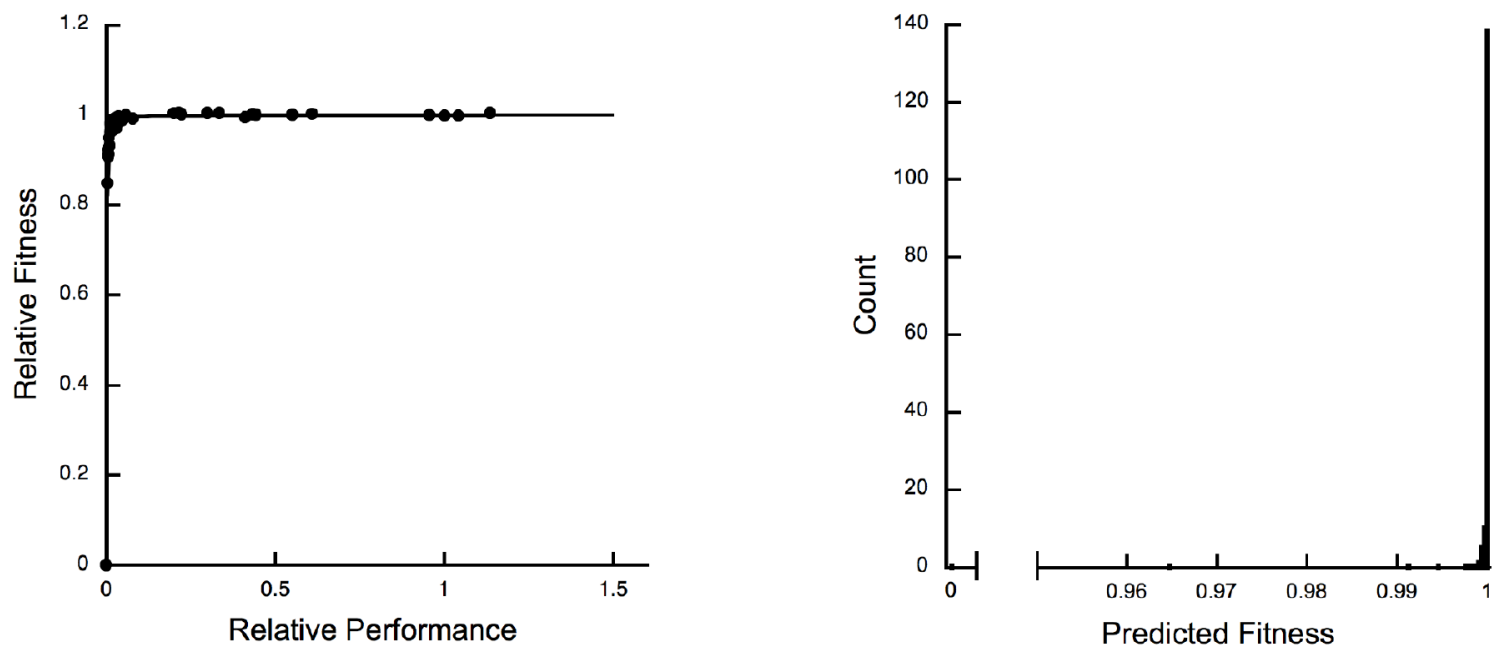

Figure 4. Large changes in IMDH performance cause small changes in fitness. (A) Relationship between fitness and performance, which conforms to a dominance curve [12], shows that E. coli wild-type IMDH lies far into a fitness plateau [58,62]. (B) As a consequence, even large reductions in enzyme activity are predicted to have very small fitness effects. The predicted fitnesses were calculated using a hyperbola, $y=1.0005 x /$ $(0.00032+x)$, fitted to the data in $(A)$.

doi:10.1371/journal.pgen.1001162.g004

potentially deleterious mutation never arose before a compensatory mutation in the same lineage.

\section{Implications of Cryptic Epistasis for Molecular Evolution}

Our demonstration of rampant cryptic epistasis in IMDH is entirely in accord with a recent insightful analysis of protein evolution that invoked extensive epistasis to account for the retarded divergence seen in ancient proteins [66]. There the case was made for a rugged fitness landscape characterized by multidimensional sign epistasis that forces sites to be conserved for billions of years until the right combination of amino acids at other sites to allows them to evolve. Our failure to identify compensatory mutations for A94D and A284C is indicative of multidimensional sign epistasis. That a single replacement is sufficient to compensate the F73L mutation demonstrates that epistasis need not always be multidimensional, however.

In an earlier study [67], a mutant library in which 52 natural amino acid replacements from 15 subtilisin orthologues had been recombined was screened for function. Sequence comparisons of the unscreened and the screened libraries suggested that almost all possible pair-wise combinations of amino acids can coexist and that functional co-dependencies are rare. These conclusions seemingly stand in contradiction to ours.

The subtilisin experiment suggests that 7 of $52 \times 51 / 2=1326$ pairs compromise function for $p=7 / 1326=0.0053$. In other words about a half percent of pair-wise interactions are deleterious. For $E$. coli $\mathrm{IMDH}$, the probability, $f$, that introducing an amino acid from an orthologue has no effect on function is $f=(1-p)^{D-1}$, where $D$ is the number of residues that differ between the two sequences. For $E$. coli IMDH we have $f=(168-63) / 168, D=168$ and hence $p=0.0028$. The two-fold difference between the two estimates of $p$ is small considering the differences between the enzymes and the experimental methods employed. The take-home lesson is that epistatic interactions may be rare individually, but their cumulative impact on evolution rapidly increases with divergence, $D$. The phenomenon is akin to the snowball effect describing the accumulation of DobzhanskiMuller incompatibilities during speciation [68].

The simplest model of sequence evolution is a Poisson process in which each site accumulates mutations at a constant rate $\lambda$. The expected number of mutations accumulated at time $t$ is simply $\lambda t$ and the variance in the number of substitutions at time $t$ is also $\lambda t$. This gives the Poisson molecular clock a characteristic variance to mean ratio of $R(t)=1$. However, sequence analyses show that the molecular clock is the over-dispersed with $R(t)>1$ [69-75]. Various hypotheses have been proposed to explain this overdispersion including episodic bursts of selection, increased rates of fixation of deleterious alleles during population bottlenecks, fluctuating neutral spaces and variable mutation rates $[73,76-$ 84]. Cryptic epistasis, in causing constraints at sites to vary and hence substitution rates at sites to vary, undoubtedly contributes to over-dispersion in the molecular clock.

Simulations show that ignoring changes in substitution rates (heterotachy) can induce systematic errors in phylogenetic reconstruction, including topological inaccuracies, long-branch biases and other effects [85-91]. Simulations also show that ignoring co-dependencies among sites causes the amount of evolution to be underestimated, particularly on branches deep in a tree [92]. The resulting impression of rapid ancient radiations with an indeterminate branching order makes identifying the origins of some taxonomic groups difficult [93,94]. Taking explicit account of co-dependencies within data has been shown to aid phylogenetic inference $[92,95]$. While recent advances accommodate temporal variability in substitution rates within sites [87], even going so far as to model pair-wise interactions between sites in close proximity using predefined statistical potentials calculated from structural data [88], general phylogenetic practice does not [89]. The extensive cryptic epistasis we have revealed suggests that the usual practice of ignoring co-dependencies among sites needs reconsidering.

Ancestral sequence resurrection is a popular experimental approach to explore ancient phenotypes and adaptations [1]. 


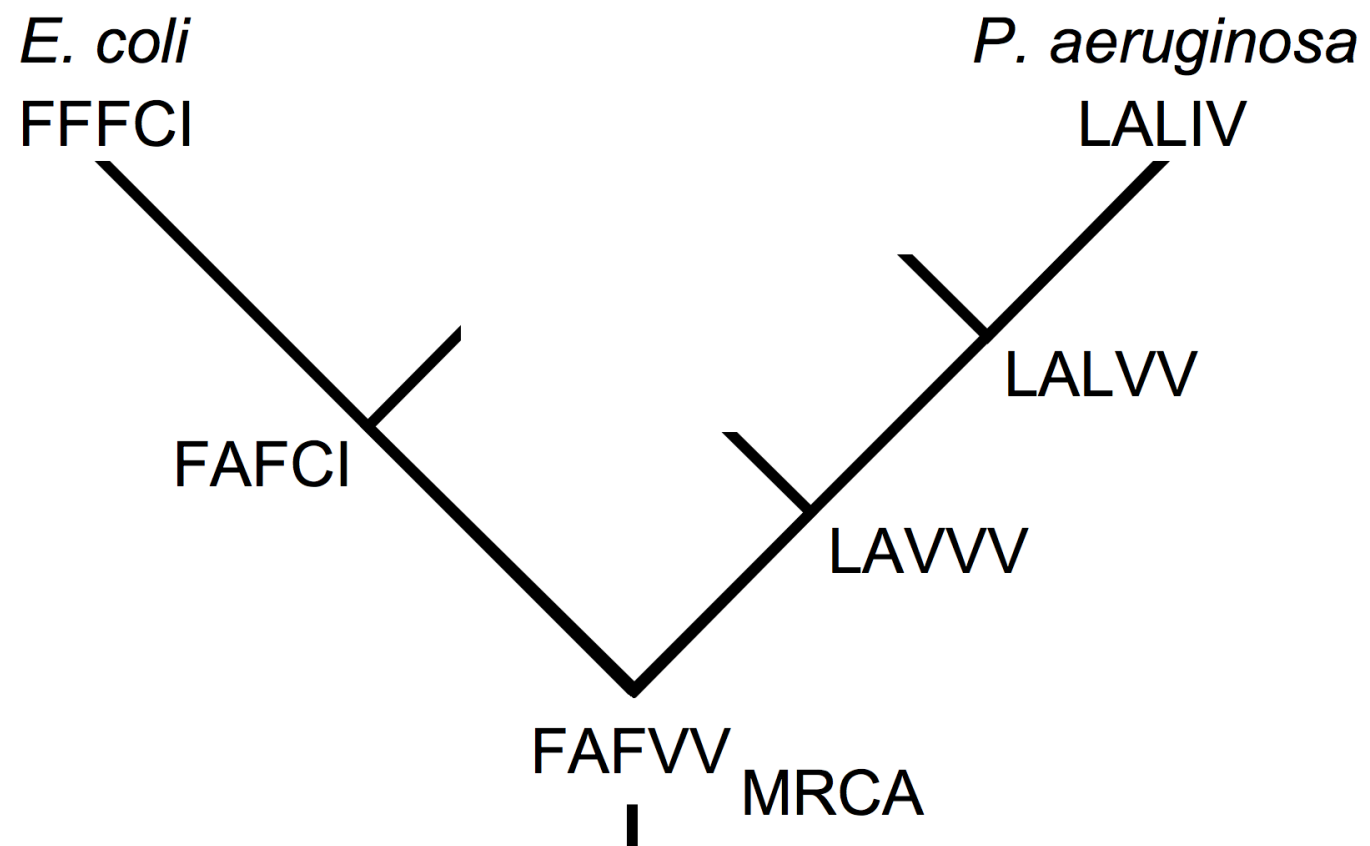

F. Incompatible mutations arise in different lineages. Each genotype is designated by five amino arranged in order of sites 73,120,132, 136 and 179. Three mutations on the lineage leading from the MCRA (defined using Geobacter metallireducens as the outgroup) to $E$. coli are needed to complete the interaction: A120F, V136C and V179I. Genotype FAFCl on the E. coli lineage and engineered into E. coli IMDH is as active as wild-type. Neither F132V nor C136V affect the performance of E. coli IMDH. Simplified from a full tree of 537 taxa. doi:10.1371/journal.pgen.1001162.g005

Accurately inferred ancestral sequences are essential, otherwise there can be little confidence in the experimental results. Caution is warranted when interpreting functional patterns that mimic in silico reconstruction biases [96]. Current methods ignore functional co-dependencies among sites; the consequences for the accuracy of inferred ancestral sequences is largely unexplored. On the one hand, coupling between sites represents a loss of degrees of freedom (knowing the residue at one site allows inferences to be made about the residues at coupled sites) that leads to overconfidence in reconstructed trees [97]. This is particularly problematic if attempting to reconstruct ancestral sequences during a supposed rapid ancient radiation. On the other hand, the same loss of degrees of freedom means that fewer inferences are made, which should improve accuracy. Simulations suggest that the conditions producing phylogenetic uncertainty also make the ancestral state identical across plausible trees [98]. This helps make ancestral sequence reconstructions robust to phylogenetic uncertainty.

Our dissection of epistatic interactions with site 73 shows that amino acid replacements accumulated during evolution can interact without affecting protein function. Nevertheless, cryptic epistasis may impact functional evolution. Reconstructing ancestral proteins on either side of an ancient functional change neglects epistatic interactions that earlier prevented the change and that later prevented the new function reverting [99] or changing in response to a new selective pressure. Such canalizing epistasis both retards functional evolution and thwarts attempts to engineer enzymes rationally [100]. Protein breeding experiments commonly use mutant libraries, generated either by recombining related sequences [101] or by allowing sequences to accumulate 'neutral drift' mutations [102], to circumvent the canalizing effects of cryptic epistasis. We speculate that the rampant cryptic epistasis, inferred by computational methods [66] and detected in experiments on IMDH, might be sufficiently extensive to resist functional changes on evolutionary time scales. Only when rare neutral mutations relieve its canalizing effects can new functions evolve. This model potentially explains why protein evolution is characterized by long periods of functional stasis punctuated by rapid functional shifts.

\section{Materials and Methods}

\section{Strains, Media, and Chemicals}

E. coli K12 strains MG1655, JW5807 (Keio Collection) [103], MM294D and BL21-gold-DleuB::kan ${ }^{r}$ have been previously described [53,58]. A derivative of $E$. coli strain BL21-gold (Stratagene) was constructed by P1 transduction [104] of the $\triangle$ leuB-leuC::kan ${ }^{r}$ construct from strain JW5807. LB medium was 
supplemented with $15 \mathrm{~g} / \mathrm{l}$ Bacto agar for plates [104]. TALON Superflow metal affinity resin and TALON xTractor Buffer were purchased from Takara Bio USA (Madison, WI). Unless specified otherwise, chemicals were purchased from Sigma-Aldrich (St. Louis) and restriction enzymes were purchased from New England Biolabs (Ipswich, MA) and Fermentas (Canada). DL-threo-3isopropylmalic acid was purchased from Wako Pure Chemical Industries (Japan).

\section{Sequencing}

All mutants sequenced at the BioMedical Genomics Center, University of Minnesota.

\section{Constructing Plasmid pLeuB}

The leu operon, from mid leuA through leu $C$, was acquired by genomic PCR from MG1655. The genomic PCR product and pMML22KBA-KYVY [58] were digested with restriction enzymes RsrII and SphI. The vector and insert were ligated by quick ligation (Fermentas), to create pLeuB7. The construct was transformed by $\mathrm{RbCl}$ transformation [105] into MM294D and selected on $\mathrm{LB} / \mathrm{Amp}(100 \mu \mathrm{g} / \mathrm{ml})$, overnight at $37^{\circ} \mathrm{C}$.

\section{Primer Design}

The $5^{\prime}$-primer is designed with $15-20$ bases, then the bases to be mutated, followed by a minimum of 12 bases at the $3^{\prime}$ end. The $3^{\prime}$-primer is complementary to the first $15-20$ bases $5^{\prime}$-primer. Thus, the primers are staggered and only the $5^{\prime}$-primer encodes the mutations to be introduced.

\section{Plasmid Methylation}

Cytosine residues in plasmid pLeuB7 were methylated by the CpG Methyltransferase M.SssI (New England Biolabs) according to the manufacture's instructions [106]. Methylated DNA and a nonmethylated control were diluted 1:25, and $2 \mu \mathrm{l}$ transformed into $E$. coli strain MM294D by using the $\mathrm{RbCl} / \mathrm{CaCl}_{2}$ method [105]. After transformation cells were plated on LB/ampicillin $(100 \mu \mathrm{g} / \mathrm{ml})$ and incubated overnight at $37^{\circ} \mathrm{C}$.

\section{Mutagenesis}

Restriction sites (Figure S5) and the 168 single mutants were introduced into wild-type $E$. coli leuB using the protocol in Table 2 [107]. Five microliters of each finished reaction was run on a $1 \%$ agarose gel to verify the PCR worked, and $2 \mu \mathrm{l}$ was transformed [105] into MM294D, plated on LB/ampicillin $(100 \mu \mathrm{g} / \mathrm{ml})$ and incubated overnight at $37^{\circ} \mathrm{C}$. The presence of mutations was confirmed by sequencing. Mutant enzymes with kinetic characteristics different from wild-type had their entire $l e u B$ gene resequenced to confirm that no other mutations had inadvertently been introduced.

Double mutants incorporating F73L, A94D and A284C with other mutations were constructed by restriction digestion and ligation using strain MM294D as a host. F73L, A94D, A284C were restriction digested and inserts with $\mathrm{L} 24 \mathrm{~V}, \mathrm{~S} 156 \mathrm{E}$, and Y360A ligated to form parent vectors F73L,L24V, F73L,S156E, A94D,L24V, A94D,S156E, A284C,S156E, and A284C,Y360A. L24V removes the AflII site, S156E removes the BamHI site, and Y360A removes the SnaBI site. Parent vectors were then restriction digested and inserts, obtained from restriction digests of other single mutants, were ligated in. After transformation [105] cells were plated on $\mathrm{LB} /$ ampicillin $(100 \mu \mathrm{g} / \mathrm{ml})$ and incubated overnight at $37^{\circ} \mathrm{C}$. Colonies were grown in $\mathrm{LB} /$ ampicillin $(100 \mu \mathrm{g} / \mathrm{ml})$ and the plasmids purified. Double mutants were identified by the presence of a restored AflII, BamHI or SnaBI restriction site. Those remaining mutations close to F73L, A94D and A284C that could not be introduced by restriction digestion and ligation were introduced by PCR mutagenesis and the entire gene sequenced.

In all 694 mutants were constructed: 17 restriction sites were introduced into pLeuB7, 170 single mutants were made (the exact position of one single amino acid deletion could not be reliably identified and so three mutates deleting residues 150,151 and 152, were constructed), and $3 \times 169$ double mutants were made.

\section{Protein Expression}

Mutant IMDHs were over-expressed from plasmids in a derivative of $E$. coli strain BL21-gold (Stratagene) formed by $\mathrm{P} 1$

Table 2. PCR reaction mixture and cycling protocol.

\begin{tabular}{|c|c|c|}
\hline \multicolumn{3}{|c|}{ Reaction mixture } \\
\hline \multicolumn{3}{|l|}{$\mu \mathbf{I}$} \\
\hline 2.0 & $\mathrm{H}_{2} \mathrm{O}$ & \\
\hline 1.0 & $10 \times$ Hot Start Buffer (Novagen) & \\
\hline 0.6 & $25 \mathrm{mM} \mathrm{MgSO}_{4}$ & \\
\hline 1.0 & 2 mM dNTP's & \\
\hline 4.0 & Methylated pLeuB7 template $(\sim 80 \mathrm{ng} / \mu \mathrm{l})$ & \\
\hline 0.6 & 5'-Primer $(0.3 \mu \mathrm{M})$ & \\
\hline 0.6 & $3^{\prime}$-Primer $(0.3 \mu \mathrm{M})$ & \\
\hline 0.2 & KOD Hot Start (Novagen) & \\
\hline \multicolumn{3}{|l|}{10.0} \\
\hline \multicolumn{3}{|c|}{ Cycling protocol repeated 20 times } \\
\hline $95^{\circ} \mathrm{C}$ & 2 mins & Polymerase Activation \\
\hline $95^{\circ} \mathrm{C}$ & 20 secs & Denaturation \\
\hline $60^{\circ} \mathrm{C}$ & 10 secs & Primer Annealing \\
\hline $70^{\circ} \mathrm{C}$ & 3 mins & 0.5 min/kb Extension \\
\hline
\end{tabular}


transduction [104] of the $\Delta l e u B-l e u C:: k^{r}{ }^{r}$ construct from strain JW5807. Transformed cells were grown overnight at $37^{\circ} \mathrm{C}$ in $5 \mathrm{~mL}$ of LB containing ampicillin $(100 \mu \mathrm{g} / \mathrm{ml})$ and $0.2 \mathrm{mM}$ IPTG. Following centrifugation, cells were resuspended in $1 \mathrm{~mL}$ of $\mathrm{BD}$ TALON xTractor Buffer (Becton-Dickenson). After $10 \mathrm{~min}$ rocking at room temperature, the sample was then centrifuged for $20 \mathrm{~min}$ at $11,200 \times \mathrm{g}$ and the supernatant transferred to a TALON $2 \mathrm{~mL}$ disposable gravity column containing $2 \mathrm{~mL}$ of equilibrated BD TALON Superflow metal affinity resin. The protein was then eluted following the manufacture's protocol with the exception that potassium salts were substituted for sodium salts. All enzymes were purified to homogeneity as judged using Coomassie stained SDS-PAGE gels.

\section{Screening Double Mutants}

Double mutants were screened for compensatory mutations at $37^{\circ} \mathrm{C}$ in $25 \mathrm{mM}$ MOPS, $100 \mathrm{mM} \mathrm{KCl}, 1 \mathrm{mM}$ DTT, pH 7.3 in the presence of fixed concentrations of $0.2 \mathrm{mM}$ DL-threo-3isopropylmalic acid and $5 \mathrm{mM} \mathrm{MgCl}_{2}$ and $0.1 \mathrm{mM} \mathrm{NAD}$. The concentration of NAD lies far below the $K_{m}$ s of the single mutants

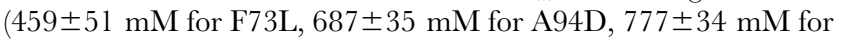
A284C). With each mutant unsaturated the rate of the reaction is proportional to $k_{c a t} / K_{m}$ making improvements in performance readily detectable.

\section{Enzyme Kinetics}

Kinetics were performed at $37^{\circ} \mathrm{C}$ in $25 \mathrm{mM}$ MOPS, $100 \mathrm{mM}$ $\mathrm{KCl}, 1 \mathrm{mM}$ DTT, pH 7.3 in the presence of fixed concentrations of $0.2 \mathrm{mM}$ DL-threo-3-isopropylmalic acid and $5 \mathrm{mM} \mathrm{MgCl}_{2}$, and with concentrations of NAD varied from $1 / 4$ to $10 \times$ the apparent $K_{m}$. Reactions were initiated by adding $10 \mu \mathrm{L}$ of mutant IMDH (diluted in $50 \mathrm{mM}$ potassium phosphate, $300 \mathrm{mM} \mathrm{KCl}, 150 \mathrm{mM}$ imidazole, $10 \mathrm{mM} \beta$-mercaptoethanol, $\mathrm{pH} \mathrm{7.0)}$ to $1 \mathrm{ml}$ of the reaction mix in a $1 \mathrm{~cm}$ semi-UV (methylacrylate) cuvette (Fisher Scientific). Reaction rates were determined spectrophotometrically by measuring the production of NADH at $340 \mathrm{~nm}$ using a molar extinction coefficient of $6220 \mathrm{M}^{-1} \mathrm{~cm}^{-1}$, in a thermostated Cary 300 Bio with a $6 \times 6$ Peltier block (Varian). Inhibition constants were determined in the presence of varying fixed concentrations of reduced coenzyme. Kinetic parameters $V_{\max }, K_{m}$ and $V_{\max } / K_{m}$ were determined using nonlinear regression as implemented in JMP (SAS Institute). Maximum turnover rates, $k_{\text {cat }}=V_{\max } /[E]$, were calculated with enzyme concentrations, $[E]$, determined spectrophotometrically by Bradford assay [108] (Bio-Rad) using bovine IgG as the standard.

Each single mutant was independently expressed, purified and kinetically characterized twice.

\section{Phylogenetics}

A total of 537 amino acid sequences (downloaded from GenBank via the NCBI web site http://www.ncbi.nlm.nih.gov/) were aligned using ClustalW software [109]. X-ray structures (IMDHs 1HEX, 1CNZ, 1CM7, IV53, 1W0D, 1WPW, 1VLC, and 1A05) were downloaded from the PDB web site (http://www. pdb.org/pdb/home/home.do) and superimposed using Swiss-Pdb Viewer software [110]. Superpositioned structures were used as a guide to adjust the alignments of highly divergent sequences. A bootstrapped neighbor joining tree was constructed with PHYLIP [111] using a JTT [112] substitution matrix with deep branches swapped and assessed by maximum likelihood. A consensus tree was generated with Mr.Bayes [113] based on a gamma distributed, mixed model of amino acid evolution. The MCMC was run for 75000 generations sampling every 50 generations with a burn-in of 500. Both trees produced similar results when ancestral sites were reconstructed by fastml [114]. With Bayesian posterior probabilities $<0.9$ accounting for $<15 \%$ of sites (mostly in flexible loops), the amino acid identities at most sites in the deduced sequence of the most recent common ancestor are reliably inferred.

\section{Supporting Information}

Figure S1 IMDH alignments. E. coli and $P$. aeruginosa IMDHs aligned with their most recent common ancestor (MRCA). Single letter amino acid code with dashes for deletions and question marks in the MRCA when Bayesian posterior probabilities fall below $90 \%$. Top numbering refers to the alignment. Bottom numbering refers to E. coli $\mathrm{IMDH}$. Asterisks above the alignment denote residues identical in both species. Protein secondary structures, $\mathrm{H}$ for helices and $\mathrm{S}$ for sheets, are indicated below the alignment.

Found at: doi:10.1371/journal.pgen.1001162.s001 (0.03 MB DOC)

Figure S2 The evolution of cryptic epistasis. (A) Moving either amino acid from one species into the enzyme of another species risks loss of function when three mutations have occurred, one at each site in one lineage and one at either site in the other lineage because genotypes $\mathrm{AB}$ and $\mathrm{aB}^{\prime}$ are each synthetic combinations. (B) Moving amino acids species B into species A identifies two sites of three engaged in pair-wise interactions (mutants $\mathrm{ABC}$ and $\mathrm{aBC}$ are synthetic combinations; mutant Abc represents an ancestral functional state).

Found at: doi:10.1371/journal.pgen.1001162.s002 (0.08 MB TIF)

Figure S3 Mutations affecting kinetic performance are distributed throughout the IMDH structure. Performance scale: red $<1 \%<$ dark pink $<10 \%<$ light pink $<50 \%<$ white $<80 \%$ of $E$. coli wildtype IMDH. F120A, the only mutation to show increased activity, is shown in green. Asp251 is at the catalytic center of the active site. The homodimer interface, a four helix bundle, is formed with the two helices on the right that have no mutations in them.

Found at: doi:10.1371/journal.pgen.1001162.s003 (3.86 MB TIF)

Figure S4 The position of F73 in IMDH. The side chain of IMDH residue $\mathrm{F} 73$ forms the side of a pocket into which the amide of the nicotiamide ring must bind during catalysis. F73 is replaced by $\mathrm{K} 100$, which is essential to catalysis in the related isocitrate dehydrogenase [53]. Coenzyme and substrate modeled into E. coli IMDH (pdb 1CM7) [53] from E. coli isocitrate dehydrogenase (pdb 1AI2).

Found at: doi:10.1371/journal.pgen.1001162.s004 (8.14 MB TIF)

Figure S5 Restriction sites introduced into wildtype $E$. coli leuB are silent substitutions. Single cut sites in bold, double cut sites (one cut elsewhere in pLeuB7) in italics.

Found at: doi:10.1371/journal.pgen.1001162.s005 (0.03 MB DOC)

\section{Acknowledgments}

We wish to thank two anonymous reviewers for their thoughtful criticisms and constructive suggestions. AMD acknowledges GVE's matchless criticisms and practiced oversight.

\section{Author Contributions}

Conceived and designed the experiments: GBG AMD. Performed the experiments: ML. Analyzed the data: ML GBG AMD. Wrote the paper: GBG AMD. 


\section{References}

1. Dean AM, Thornton JW (2007) Mechanistic approaches to the study of evolution: the functional synthesis. Nat Rev Genet 8: 675-688.

2. Felsenstein J (2004) Inferring phylogenies. Sunderland: Sinaur Assoc Inc.

3. Nei M, Kumar S (2000) Molecular evolution and phylogenetics. Oxford: Oxford University Press.

4. Smith NG, Eyre-Walker A (2002) Adaptive protein evolution in Drosophila. Nature 415: 1022-1024.

5. Charlesworth J, Eyre-Walker A (2006) The rate of adaptive evolution in enteric bacteria. Mol Biol Evol 23: 1348-1356.

6. Sawyer SA, Kulathinal RJ, Bustamante CD, Hartl DL (2003) Bayesian analysis suggests that most amino acid replacements in Drosophila are driven by positive selection. J Mol Evol 57: 154-164.

7. Hughes AL (2008) Near neutrality: leading edge of the neutral theory of molecular evolution. Ann NY Acad Sci 1133: 162-179.

8. DePristo MA, Weinreich DM, Hartl DL (2007) Missense meanderings in sequences space: a biophysical view of protein evolution. Nat Rev Genet 6 : 678-687.

9. Presgraves DC (2010) The molecular evolutionary basis of species formation. Nat Rev Genet 11: 175-180.

10. Barton NH, Charlesworth B (1998) Why sex and recombination? Science 281: 1986-1990.

11. Kondrashov AS (1988) Deleterious mutations and the evolution of sexual reproduction. Nature 336: 435-440.

12. Kacser H, Burns JA (1981) The molecular basis of dominance. Genetics 97: 639-666.

13. Jasnos L, Korona M (2007) Epistatic buffering of fitness loss in yeast double deletion strains. Nat Genet 39: 550-554.

14. Cordell HJ (2009) Detecting gene-gene interactions that underlie human diseases. Nat Rev Genet 10: 392-404.

15. Zuckerkandl E (1963) Perspectives in molecular anthropology. In: Washburn SL, ed. Classification and human evolution. Chicago: Aldine. pp 243-272.

16. Fitch WM, Markowitz E (1970) An improved method for determining codon variability in a gene and its application to the rate of fixation of mutations in evolution. Biochem Genet 4: 579-593.

17. Korber BT, Farber RM, Wolpert DH, Lapedes AS (1993) Covariation of mutations in the V3 loop of human immunodeficiency virus type 1 envelope protein: an information theoretic analysis. Proc Natl Acad Sci USA 90: 7176-7180.

18. Göbel U, Sander G, Schneider R, Valencia (1994) A Correlated mutations and residue contacts in proteins. Proteins 18: 309-317.

19. Shindyalov IN, Kolchanov NA, Sander C (1994) Can three-dimensional contacts in protein structures be predicted by analysis of correlated mutations? Protein Eng 7: 349-358.

20. Taylor WR, Flores TP, Orengo CA (1994) Multiple protein structure alignment. Protein Sci 3: 1858-1870.

21. Lockless SW, Ranganathan R (1999) Evolutionarily conserved pathways of energetic connectivity in protein families. Science 286: 295-299.

22. Pritchard L, Bladon PMO, Mitchell JJ, Dufton M (2001) Evaluation of a novel method for the identification of coevolving protein residues. Protein Eng 14: 549-555.

23. Valdar WS (2002) Scoring residue conservation. Proteins 48: 227-241.

24. Süel GM, Lockless SW, Wall MA, Ranganathan R (2003) Evolutionarily conserved networks of residues mediate allosteric communication in proteins. Nat Struct Biol 10: 59-69.

25. Socolich M, Lockless SW, Russ WP, Lee H, Gardner KH, et al. (2005) Evolutionary information for specifying a protein fold. Nature 437: 512-518.

26. Russ WP, Lowery DM, Mishra P, Yaffe MB, Ranganathan R (2005) Naturallike function in artificial WW domains. Nature 437: 579-583.

27. Gloor GB, Martin LC, Wahl LM, Dunn SD (2005) Mutual information in protein multiple sequence alignments reveals two classes of coevolving positions. Biochemistry 44: 7156-7165.

28. Ané C, Burleigh JG, McMahon MM, Sanderson MJ (2005) Covarion structure in plastid genome evolution: a new statistical test. Mol Biol Evol 22: 914-924.

29. Wang K, Samudrala R (2006) Incorporating background frequency improves entropy- based residue conservation measures. BMC Bioinformatics 17: 385.

30. Tuffley C, Steel MA (1997) Modelling the covarion hypothesis of nucleotide substitution. Math Biosci 147: 63-91.

31. Lockhart PJ, Steel MA, Barbrook AC, Huson DH, Charleston MA, et al. (1998) A covariotide model explains apparent phylogenetic structure of oxygenic photosynthetic lineages. Mol Biol Evol 15: 1183-1188.

32. Pollock DD, Taylor WR, Goldman N (1999) Coevolving protein residues: Maximum likelihood identification and relationship to structure. J Mol Biol 287: 187-198 (1999).

33. Galtier N (2001) Maximum-likelihood phylogenetic analysis under a covarionlike model. Mol Biol Evol 18: 866-873.

34. Huelsenbeck JP (2002) Testing a covariotide model of DNA substitution. Mol Biol Evol 19: 698-707.

35. Dutheil J, Pupko T, Jean-Marie A, Galtier N (2005) A model-based approach for detecting coevolving positions in a molecule. Mol Biol Evol 22: 1919-1928.
36. Fares MA, Travers SAA (2006) A Novel Method for detecting intramolecular coevolution: Adding a further dimension to selective constraints analyses. Genetics 173: 9-23.

37. Wang HC, Spencer M, Susko E, Roger AJ (2007) Testing for covarion-like evolution in protein sequences. Mol Biol Evol 24: 294-305.

38. Rodrigue N, Kleinman CL, Philippe H, Lartillot N (2009) Computational methods for evaluating phylogenetic models of coding sequence evolution with dependence between codons. Mol Biol Evol 26: 1663-1676.

39. Dean AM, Golding GB (2000) Enzyme evolution explained (sort of). In Altman RB, Dunker AK, Hunter L, Lauderdale K, Klein TE, eds. The Pacific symposium on bioinformatics 2000: Singapore: World Scientific.

40. Dean AM, Neuhauser C, Grenier C, Golding GB (2002) The pattern of amino acid replacements in $\alpha / \beta$-barrels. Mol Biol Evol 19: 1846-1864.

41. Kondrashov AS, Sunyaev S, Kondrashov FA (2002) Dobzhansky-Muller incompatibilities in protein evolution. Proc Natl Acad Sci USA 99: 14878-14883.

42. Kulathinal RJ, Bettencourt BR, Hartl DL (2004) Compensated deleterious mutations in insect genomes. Science 306: 1553-1554.

43. Gloor GB, Tyagi G, Abrassart DM, Kingston AJ, Fernandes AD, et al. (2010) Functionally compensating, coevolving positions are neither homoplasic nor conserved in clades. Mol Biol Evol 27: 1181-1191.

44. Fisher A, Shi Y, Ritter A, Ferretti JA, Perez-Lamboy G, et al. (2000) Functional correlation in amino acid residue mutations of yeast iso-2-cytochrome $c$ that is consistent with the prediction of the concomitantly variable codon theory in cytochrome $c$ evolution. Biochem Genet 38: 181-200.

45. Malcolm BA, Wilson KP, Matthews BW, Kirsch JF, Wilson AC (1990) Ancestral lysozymes reconstructed, neutrality tested, and thermostability linked to hydrocarbon packing. Nature 345: 86-89.

46. Wilson KP, Malcom BA, Matthews BW (1992) Structural and thermodynamic analysis of compensating mutations within the core of chicken egg white lysozyme. J Biol Chem 267: 10842-10849.

47. Mateu MG, Fersht A (1999) Mutually compensatory mutations during evolution of the tetramerization domain of tumor suppressor p53 lead to impaired hetero-oligomerization. Proc Natl Acad Sci USA 96: 3595-3599.

48. Weinreich DM, Delaney NF, DePristo MA, Hartl DL (2006) Darwinian evolution can follow only very mutational paths to fitter proteins. Science 312: $111-114$.

49. Bridgham JT, Carroll SM, Thornton JW (2006) Evolution of hormonereceptor complexity by molecular exploitation. Science 312: 97-101.

50. Yokoyama S, Tada T, Zhang H, Britt L (2008) Elucidation of phenotypic adaptations: molecular analyses of dim-light vision proteins in vertebrates. Proc Natl Acad Sci USA 105: 13480-13485.

51. Field SF, Matz MV (2010) Retracing evolution of red fluorescence in GFP-like proteins from Faviina corals. Mol Biol Evol 27: 225-233.

52. Stryer L (1995) Biochemistry. New York: W. H. Freeman \& Co.

53. Miller SP, Lunzer M, Dean AM (2006) Direct demonstration of an adaptive constraint. Science 314: 458-461.

54. Imada K, Sato M, Tanaka N, Katsube Y, Matsuura Y, et al. (1991) Threedimensional structure of a highly thermostable enzyme, 3-isopropylmalate dehydrogenase of Thermus thermophilus at $2.2 \AA$ resolution. J Mol Biol 222: 725-738.

55. Wallon G, Kryger G, Lovett ST, Oshima T, Ringe D, et al. (1997) Crystal structures of Escherichia coli and Salmonella typhimurium 3-isopropylmalate dehydrogenase and comparison with their thermophilic counterpart from Thermus thermophilus. J Mol Biol 266: 1016-1031.

56. Imada K, Inagaki K, Matsunami H, Kawaguchi H, Tanaka H, et al. (1998) Structure of 3- isopropylmalate dehydrogenase in complex with 3-isopropylmalate at $2.0 \AA$ resolution: the role of Glu88 in the unique substraterecognition mechanism. Structure 6: 971-982.

57. Tsuchiya D, Sekiguchi T, Takenaka A (1997) Crystal structure of 3isopropylmalate dehydrogenase from the moderate facultative thermophile, Bacillus coagulans: two strategies for thermostabilization of protein structures. J Biochem (Tokyo) 122: 1092-104.

58. Lunzer M, Miller SP, Felsheim R, Dean AM (2005) The biochemical architecture of an ancient adaptive landscape. Science 310: 499-501.

59. Sokal RR, Rohlf FJ (1995) Biometry: The Principles and Practices of Statistics in Biological Research, $3^{\text {rd }}$ ed. NY: WH Freeman.

60. Benjamini Y, Hochberg Y (1995) Controlling the false discovery rate: a practical and powerful approach to multiple testing. J Roy Statist Soc B 57: 289-300.

61. Fersht A (1998) Structure and Mechanism in Protein Science: A Guide to Enzyme Catalysis and Protein Folding. NY: WH Freeman.

62. Hartl DL, Dykhuizen DE, Dean AM (1985) Limits of adaptation: the evolution of selective neutrality. Genetics 111: 655-674.

63. Kuiken KA, Lyman CM (1948) The availability of amino acids in some foods. J Nutr 36: 359-368.

64. Fitch WM, Markowitz E (1970) An improved method for determining codon variability in a gene and its application to the rate of fixation of mutations in evolution. Biochem Genet 4: 579-593.

65. Ohta T (1992) The nearly neutral theory of molecular evolution. Annu Rev Ecol Syst 23: 263-286. 
66. Povolotskaya IS, Kondrashov FA (2010) Sequence space and the ongoing expansion of the protein universe. Nature 465: 922-927.

67. Govindarajan S, Ness JE, Kim S, Mundorff EC, Minshull J, et al. (2003) Systematic variation of amino acid substitutions for stringent assessment of pairwise covariation. J Mol Biol 328: 1061-1069.

68. Orr HA (1995) The population genetics of speciation: the evolution of hybrid incompatibilities. Genetics 139: 1805-1813.

69. Ohta T, Kimura M (1971) On the constancy of the evolutionary rate of cistrons. J Mol Evol 1: 18-25.

70. Langley CH, Fitch WM (1973) The constancy of evolution: a statistical analysis of $\alpha$ and $\beta$ haemoglobins, cytochrome c, and point fibrinopeptide A. In: Morton NE, ed. Genetic Structure of Populations. Honolulu: University of Hawaii Press. pp 246-262.

71. Langley CH, Fitch WM (1974) An examination of the constancy of the rate of molecular evolution. J Mol Evol 3: 161-177.

72. Gillespie JH, Langley CH (1979) Are evolutionary rates really variable? J Mol Evol 13: 27-34.

73. Gillespie JH (1991) The causes of molecular evolution. Oxford, UK: Oxford University Press.

74. Ohta T (1995) Synonymous and nonsynonymous substitutions in mammalian genes and the nearly neutral theory. J Mol Evol 40: 56-63.

75. Bedford T, Wapinski I, Hartl DL (2008) Overdispersion of the molecular clock varies between yeast, Drosophila and mammals. Genetics 179: 977-984

76. Gillespie JH (1984) The molecular clock may be an episodic clock. Proc Natl Acad Sci USA 81: 8009-8013.

77. Gillespie JH (1984) Molecular evolution over the mutational landscape. Evolution 38: 1116-1129.

78. Takahata N (1987) On the overdispersed molecular clock. Genetics 116: $169-179$

79. Ohta T, Tachida $\mathrm{T}$ (1990) Theoretical study of near neutrality. I. Heterozygosity and rate of mutant substitution. Genetics 126: 219-229.

80. Tachida H (1991) A study on a nearly neutral mutation model in finite populations. Genetics 128: 183-192.

81. Iwasa Y (1993) Overdispersed molecular evolution in constant environments. J Theor Biol 164: 373-393.

82. Takahata N (1991) Statistical models of the over-dispersed molecular clock. Theoret Popul Biol 39: 329-344.

83. Araki H, Tachida H (1997) Bottleneck effect on evolutionary rate in the nearly neutral mutation model. Genetics 147: 907-914.

84. Cutler DJ (2000) Understanding the over-dispersed molecular clock. Genetics 154: 1403-1417.

85. Kolaczkowski B, Thornton JW (2004) Performance of maximum parsimony and likelihood phylogenetics when evolution is heterogeneous. Nature 431: 980-984.

86. Gruenheit N, Lockhart PJ, Steel M, Martin W (2008) Difficulties in testing for covarion- like properties of sequences under the confounding influence of changing proportions of variable sites. Mol Biol Evol 25: 1512-1520.

87. Wang HC, Susko E, Roger AJ (2009) PROCOV: maximum likelihood estimation of protein phylogeny under covarion models and site-specific covarion patter analysis. BMC Evol Biol 9: 225

88. Rodrigue N, Kleinman CL, Philippe H, Lartillot N (2009) Computational methods for evaluating phylogenetic models of coding sequence evolution with dependence between codons. Mol Biol Evol 26: 1663-1676.

89. Felsenstein J (2004) Inferring phylogenies. Sunderland: Sinaur Assoc Inc.

90. Kolaczkowski B, Thornton JW (2008) A mixed branch length model of heterotachy improves phylogenetic accuracy. Mol Biol Evol 25: 1054-1066.
91. Kolaczkowski B, Thornton JW (2009) Long-branch attraction bias and inconsistency in Bayesian phylogenetics. PLoS ONE 4: e7891. doi:10.1371/ journal.pone.0007891.

92. Whelan S (2008) The genetic code can cause systematic bias in simple phylogenetic models. Phil Trans Roy Soc B 363: 4003-4011.

93. Rokas A, Krüger D, Carroll SB (2005) Animal evolution and the molecular signature of radiations compressed in time. Science 310: 1933-1938.

94. Rokas A, Carroll SB (2006) Bushes in the tree of life. PLoS Biol 4: e352. doi:10.1371/journal.pbio.0040352.

95. Schöniger M, von Haeseler A (1994) A stochastic model for the evolution of autocorrelated DNA sequences. Mol Phylogenet Evol 3: 240-247.

96. Williams PD, Pollock DD, Blackburne BP, Goldstein RA (2006) Assessing the accuracy of ancestral protein reconstruction methods. PLoS Comput Biol 2: e69. doi:10.1371/journal.pcbi.0020069.

97. Tillier ERM, Collins RA (1995) Neighbor Joining and Maximum Likelihood with RNA sequences: addressing the interdependence of sites. Mol Biol Evol 12: 7-15.

98. Hanson-Smith V, Kolaczkowski B, Thornton JW (2010) Robustness of ancestral sequence reconstruction to phylogenetic uncertainty. Mol Biol Evol 2010 Apr 5. [Epub ahead of print].

99. Bridgham JT, Ortlund EA, Thornton JW (2009) An epistatic ratchet constrains the direction of glucocorticoid receptor evolution. Nature 461: 515-519.

100. Tokuriki N, Stricher F, Serrano L, Tawfik DS (2008) How protein stability and new functions trade off. PLoS Comput Biol 4: e1000002. doi:10.1371/ journal.pcbi.1000002.

101. Stemmer WP (1994) Rapid evolution of a protein in vitro by DNA shuffling. Nature 370: 389-391

102. Bershtein S, Goldin K, Tawfik DS (2008) Intense neutral drifts yield robust and evolvable consensus proteins. J Mol Biol 379: 1029-1044.

103. Baba T, Ara T, Hasegawa M, Takai Y, Okumura Y, et al. (2006) Construction of Escherichia coli K-12 in-frame, single-gene knockout mutants: the Keio collection. Mol Syst Biol 2: 2006.0008.

104. Miller JH (1992) A short course in bacterial genetics. Cold Spring Harbor: Cold Spring Harbor Laboratory Press.

105. Hanahan D, Jessee J, Bloom FR (1991) Plasmid transformation of E. coli and other bacteria. Methods Enzymol 204: 63-113.

106. New England Biolabs (1994) The NEB Transcript 6: 7.

107. Novagen (2009) User protocol TB506 Rev. A 0408.

108. Bradford MM (1976) A rapid and sensitive method for the quantitation of microgram quantities of protein utilizing the principle of protein-dye binding. Anal Biochem 72: 248-254

109. Thompson JD, Higgins DG, Gibson TJ (1994) CLUSTAL W: improving the sensitivity of progressive multiple sequence alignment through sequence weighting, position- specific gap penalties and weight matrix choice. Nucleic Acids Res 22: 4673-4680.

110. Guex N, Peitsch MC (1997) SWISS-MODEL and the Swiss-PdbViewer: an environment for comparative protein modeling. Electrophoresis 18: 2714-2723.

111. Felsenstein J (1994) PHYLIP Version 3.5. Seattle: University of Washington,

112. Jones DT, Taylor WR, Thornton JM (1992) The rapid generation of mutation data matrices from protein sequences. Comp Appl Biosci 8: 275-282.

113. Ronquist F, Huelsenbeck JP (2003) MRBAYES 3: Bayesian phylogenetic inference under mixed models. Bioinformatics 19: 1572-1574.

114. Pupko T, Pe'er I, Graur D, Hasegawa M, Friedman N (2002) A branch-andbound algorithm for the inference of ancestral amino-acid sequences when the replacement rate varies among sites: application to the evolution of five gene families. Bioinformatics 18: 1116-1123. 